\title{
The Atmospheric Response to Surface Heating under Maximum Entropy Production
}

\author{
A. GJermundsen, J. H. LaCAsce, And L. S. GrafF \\ Meteorology and Oceanography Section, Department of Geosciences, University of Oslo, Oslo, Norway
}

(Manuscript received 19 June 2013, in final form 15 January 2014)

\begin{abstract}
In numerous studies, midlatitude storm tracks have been shown to shift poleward under global warming scenarios. Among the possible causes, changes in sea surface temperature (SST) have been shown to affect both the intensity and the position of the tracks. Increased SSTs can increase both the lateral heating occurring in the tropics and the midlatitude temperature gradients, both of which increase tropospheric baroclinicity.

To better understand the response to altered SST, a simplified energy balance model (EBM) is used. This employs the principal of maximum entropy production (MEP) to determine the meridional heat fluxes in the atmosphere. The model is similar to one proposed by Paltridge (1975) but represents only the atmospheric response (the surface temperatures are fixed). The model is then compared with a full atmospheric general circulation model [Community Atmosphere Model, version 3 (CAM3)].

In response to perturbed surface temperatures, EBM exhibits similar changes in (vertically integrated) air temperature, convective heat fluxes, and meridional heat transport. However, the changes in CAM3 are often more localized, particularly at low latitudes. This, in turn, results in a shift of the storm tracks in CAM3, which is largely absent in EBM. EBM is more successful, however, at representing the response to changes in highlatitude heating or cooling. Therefore, MEP is evidently a plausible representation for heat transport in the midlatitudes, but not necessarily at low latitudes.
\end{abstract}

\section{Introduction}

Storm tracks, that is, the regions of maximum transient eddy activity, play a central role in midlatitude climate over a range of time scales. Besides determining local weather and precipitation patterns, they contribute crucially to the meridional transport of heat, moisture, and momentum (e.g., Blackmon et al. 1977; Hoskins and Valdes 1990; Chang et al. 2002). There has been much interest in storm-track dynamics in recent years as the location, frequency, and intensity of midlatitude storms appear to be changing (McCabe et al. 2001; Wang et al. 2006). In particular, there is growing evidence of a poleward shift of the storm tracks. Similar changes have been seen in a range of atmospheric and climate models (Kushner et al. 2001; Yin 2005; Lorenz and DeWeaver 2007; Brayshaw et al. 2008; Lu et al. 2010; Graff and LaCasce 2012, hereafter GL12).

Nevertheless, it remains unclear why the storm tracks respond as they do. Both the mean circulation (the

\footnotetext{
Corresponding author address: Ada Gjermundsen, Meteorology and Oceanography Section, University of Oslo, P.O. Box 1022, Blindern, N-0315 Oslo, Norway.

E-mail: ada.gjermundsen@geo.uio.no
}

Hadley cells) and the eddy fluxes change in these simulations, and the response is likely a nonlinear one. Several factors have been identified as important, such as changes in the height of the tropopause (Lorenz and DeWeaver 2007), in sea surface temperature (SST) (Brayshaw et al. 2008; Lu et al. 2010; GL12), and in the static stability in the subtropics and the midlatitudes ( $\mathrm{Lu}$ et al. 2008). Changes in ozone concentration in the stratosphere have also been implicated (Polvani and Kushner 2002; Son et al. 2008), as have changes in eddy phase speeds (Chen et al. 2008). Butler et al. (2011) suggest that changes in eddy heat fluxes stemming from a steepening of the extratropical isentropic slope due under enhanced tropical heating are responsible. As of this writing, no consensus had been reached.

The shift in most cases is linked to a change in the baroclinicity of the troposphere. Hereafter, we focus on SST forcing as the driver of such a change. This is motivated in part by the observation that the oceans are indeed warming (e.g., Levitus et al. 2000). In model simulations with both aquaplanet (all ocean) and realistic (i.e., also with land) surface conditions, the storm tracks shift under SST warming scenarios. Exactly how the atmosphere responds depends on the details of the 
warming. Increasing SSTs at low latitudes increases latent heating there, causing the tropical atmosphere to warm. The Hadley cells widen and the storm tracks shift poleward with the mean zonal jets. Altering the SST gradients also affects the tracks, depending on where the gradient is strengthened or weakened. If increased at midlatitudes, the tracks intensify and shift poleward; if intensified at low latitudes, the tracks intensify but shift toward the equator instead (Brayshaw et al. 2008; $\mathrm{Lu}$ et al. 2010; GL12).

It would be valuable if similar changes could be observed in an idealized model, as this would shed light on the dominant mechanisms. Hereafter, we examine a candidate. This is an energy balance model (EBM) in which the eddy fluxes maximize entropy production (MEP). Such MEP models have been used previously to study the climate system. The first such model, proposed by Paltridge $(1975,1978)$, yielded surprisingly realistic predictions for the zonally averaged surface temperature, fractional cloud cover, and meridional heat fluxes.

Paltridge's success generated substantial interest, and a number of investigations followed. Grassl (1981) and Paltridge et al. (2007) studied the atmospheric response to a doubling of $\mathrm{CO}_{2}$. Pujol and Llebot (2000b) used a two-dimensional (in latitude and longitude) model to test different climate scenarios, obtaining reasonable results when compared to observations of the current climate (they found, however, too low sensitivity at high latitudes). Ozawa and Ohmura (1997) used an EBM to study the vertical temperature distribution and the magnitude of convective heat fluxes. Others have applied MEP models to different planetary atmospheres; the models of Lorenz et al. (2001) and Lorenz (2002) successfully predicted zonally averaged temperature differences on Mars and Titan. These successes suggest MEP-based EBMs could be used for climate sensitivity studies (Kleidon 2004, 2009).

The present goal is to see whether an MEP model can simulate the climate changes due to applied forcing. Thus, the work is similar in spirit to studies such as those of Grassl (1981) and Paltridge et al. (2007), but the focus here is on the sensitivity to SST and, in particular, on the position of the storm tracks. Of course, the MEP model has no storms. But it does have meridional heat transport, and the storm tracks coincide with the maximum in transport. Thus, the latter can be used as a proxy for the storm track position (e.g., Lucarini and Ragone 2011).

In contrast with Paltridge's original model, the present model solves for atmospheric transports alone, with surface conditions fixed. So the model is the EBM counterpart of an atmospheric general circulation model (AGCM) simulation, like those studied by Bengtsson et al. (2006), Brayshaw et al. (2008), and GL12. The forcing applied here follows that used by GL12, and the results will be compared directly to that study.

Hereafter, we discuss MEP and the rationale behind using this to determine eddy fluxes. Then we "build" an atmosphere-only EBM, which we refer to as the atmospheric MEP model (AMEP). This is a one-dimensional model (representing the zonally and vertically averaged atmosphere), with fixed surface temperatures. The model equations are determined in two steps. First, MEP is used to deduce how the convective heat fluxes depend on surface temperature. Then MEP is applied to diagnose the meridional fluxes. We subsequently assess to what extent AMEP captures shifts in the position of maximum heat fluxes and compare the results to the AGCM simulations of GL12.

\section{Maximum entropy production}

The climate system is a thermodynamic system in nonequilibrium. The net radiation is positive in hightemperature regions and negative in low-temperature regions, generating available potential energy (APE). This can be converted into kinetic energy, which then dissipates, generating entropy. As such, the climate system functions as a thermodynamic heat engine.

There have been several attempts to cast the climate system as one which adjusts to an extremal state. Lorenz $(1955,1960)$ suggested the atmospheric general circulation is in a state in which APE production is near its maximum possible rate. Several subsequent studies argued the atmospheric circulation instead maximizes entropy production (e.g., Lorenz 1955, 1960; Paltridge 1975). The principle of MEP has been applied to diverse nonequilibrium systems in statistical mechanics and is the counterpart to the maximum entropy principle for equilibrium systems (Kittel and Kroemer 1980). In fact, if the atmosphere is in a steady state and viscous heating is negligible compared to radiative heating (or cooling), the generation of APE is proportional to the entropy production; thus, maximizing one implies maximizing the other (Ozawa et al. 2003).

For nonequilibrium systems, the MEP state is proposed to be the most probable among all accessible states. But MEP remains a postulate; it has not yet been established theoretically, despite several attempts (Ziegler and Wehrli 1987; Dewar 2003). The forerunner of the concept is the principle of minimum entropy production, deduced for systems near equilibrium by Prigogine (1947; see also de Groot and Mazur 1963). However, MEP is perhaps closer in spirit though to the notion that heat transport is maximized in turbulent systems (Malkus 1954; Busse 1970; Howard 1972). As Ozawa et al. (2003) note, the interest in MEP is really in its application, as it 
appears to yield plausible temperature distributions in different climates.

The dominant contributor to entropy production in the climate system is radiation processes. At the top of the atmosphere (TOA), the outgoing entropy flux exceeds the incoming, implying entropy is produced within the system. This occurs because of irreversible processes such as vertical and lateral turbulent fluxes, which are commonly denoted as the "material entropy" (Pascale et al. 2011). Material entropy production in the system must be positive to satisfy the second law of thermodynamics. At the same time, having a steady state implies total entropy production must be zero. As such, the outgoing entropy flux at TOA must be balanced by the material entropy production in a steady-state climate. It is the material entropy production due to turbulent heat fluxes which varies and is maximized under MEP (Ozawa et al. 2003). Pascale et al. (2011) calculated the material entropy production to be approximately $50 \mathrm{~mW} \mathrm{~m}{ }^{-2} \mathrm{~K}^{-1}$. It is dominated by latent heat transfer $(\sim 70 \%)$ and by the atmospheric dissipation of kinetic energy $(\sim 25 \%)$.

Of course, the atmosphere is not in a steady state. It exhibits seasonal, annual, and decadal variations, and there is the long-term drift associated with global warming. But the associated imbalances are small in comparison to the main components, and it is traditional to assume the system is near a steady state, allowing application of principles like MEP. Thus, incoming radiation is balanced by outgoing radiation, potential energy generation is balanced by energy dissipation, and no kinetic energy is stored in the system (Lorenz 1955, 1960).

The MEP principle can be illustrated as follows. If a system with temperature $T$ is heated by an amount $d Q$, its entropy increases by

$$
d S=\frac{d Q}{T} .
$$

If we consider one warm region (e.g., the low-latitude atmosphere) and one cold region (e.g., the high latitudes), the entropy resulting from a heat flux $d F$ between the regions is

$$
\dot{S}=\frac{d S}{d t}=d F\left(\frac{1}{T_{C}}-\frac{1}{T_{W}}\right) .
$$

As the temperature in the cold region $T_{C}$ is less than that in the warmer region $T_{W}$, the entropy production is positive if $d F$ is positive (down the temperature gradient). A state of radiative equilibrium, where $d F$ is zero, corresponds to zero entropy production. A state of thermal equilibrium, or equivalently maximum heat flux, produces the same temperature in the two regions and zero entropy production as well. Thus the observed state, with a nonzero meridional heat flux and a nonzero temperature difference, lies between these two extremes. The MEP postulate is that the system adjusts to a state in which $\dot{S}$ is maximized. ${ }^{1}$

Whether the climate actually is in an MEP state remains to be demonstrated. The MEP conjecture, moreover, has been strongly criticized (Goody 2007; Caldeira 2007; Grinstein and Linsker 2007). Repeated applications currently appear to be the best way to test its validity.

\section{The atmospheric model}

We make such an application hereafter by comparing an MEP model with the output of an AGCM, subjected to prescribed SST perturbations. The AGCM is the National Center for Atmospheric Research (NCAR) Community Atmosphere Model, version 3 (CAM3). The model was run as a stand-alone version, with the Community Land Model, version 3.0, and a thermodynamic sea ice model, over a 20-yr period from 1 December 1980 to 28 February 2000. CAM3 was run in T42 resolution with 26 vertical layers. In the control run, standard surface forcing over a $20-y r$ period was used. Monthly SSTs were specified using climatological data.

GL12 conducted five sensitivity runs, in which the SSTs were altered by $2 \mathrm{~K}$ in different latitude bands. These changes are shown in Fig. 1. The specific runs were

- the " $2 \mathrm{~K}$ " run (Fig. 1b), where SSTs were increased uniformly over all oceans;

- the "2K-lowlat" run (Fig. 1a), where SSTs were increased equatorward of $45^{\circ}$;

- the "2K-tropics" run (Fig. 1d), where SSTs were increased equatorward of $15^{\circ}$; and

- the "2K-highlat" (Fig. 1c) and "-2K-highlat" runs, where SSTs where increased and decreased poleward of $45^{\circ}$, respectively.

The temperature perturbation in each case was tapered to zero over an $8^{\circ}$ latitude range. For further details, see GL12.

The fields used hereafter derive from averages over the 20-yr period. For comparison with the MEP model

\footnotetext{
${ }^{1}$ Lucarini (2009) argue that the MEP state is equivalent to a combined optimization of the down-gradient heat transport and of the production of mechanical work associated with the production (and dissipation) of kinetic energy from APE. In a simple box model, this can be understood as an optimal balance of the heat fluxes and the temperature gradients that drives the fluxes.
} 
a)

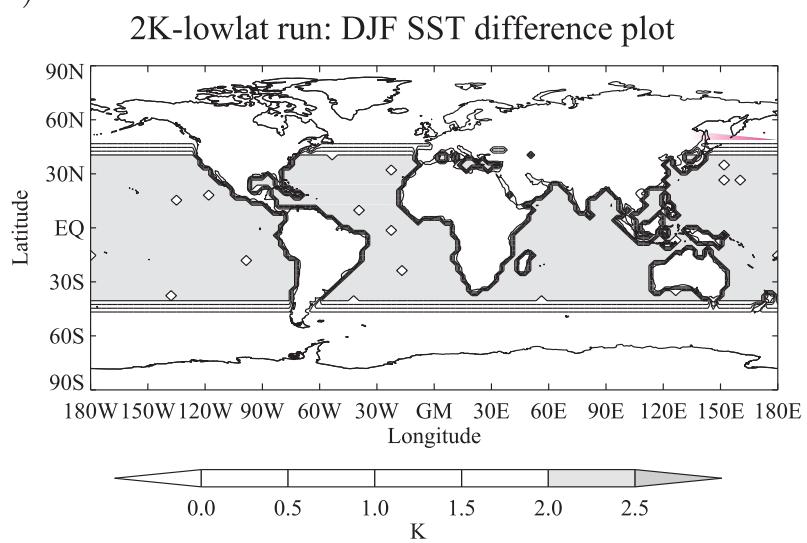

c)

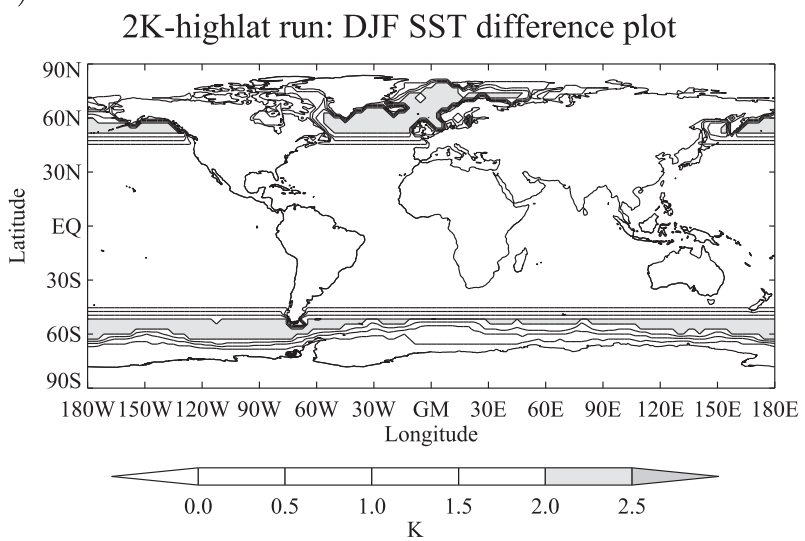

b)

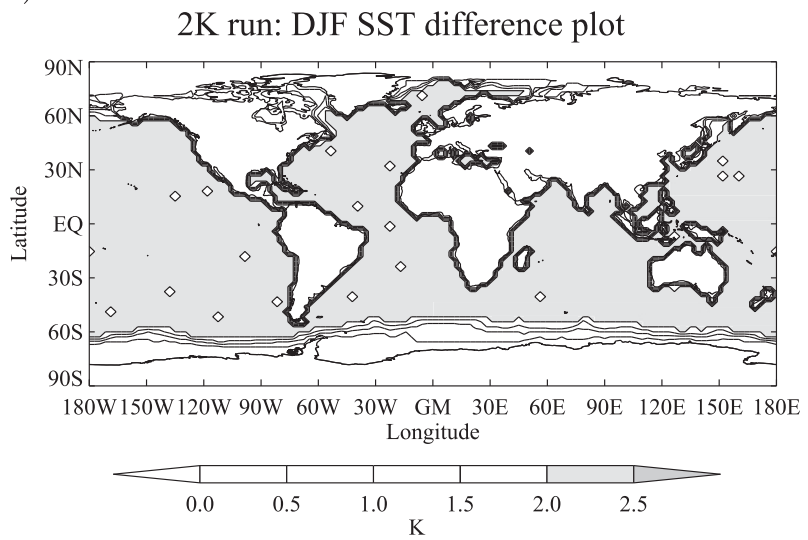

d)

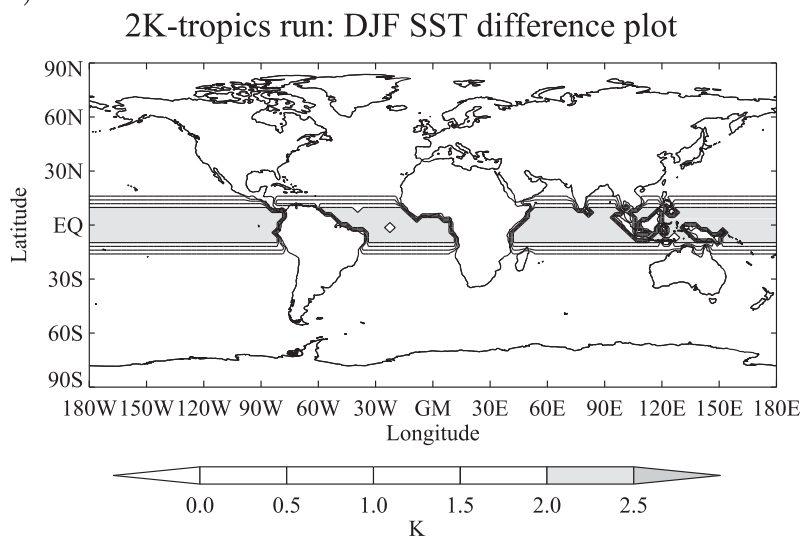

FIG. 1. The SST forcing in the (a) 2K-lowlat, (b) 2K, (c) 2K-highlat (-2K-highlat, only negative), and (d) 2K-tropics runs. Contour interval is $0.5 \mathrm{~K}$, as indicated in the gray-shaded bar. Figure from GL12.

(which has a single box in the vertical), we require a mean atmospheric temperature from CAM3. We take this to be the density-weighted mean:

$$
T \equiv \frac{\int_{V} \bar{T}(x, y, z) \bar{\rho}(x, y, z) d V}{\int_{V} \bar{\rho}(x, y, z) d V} .
$$

Here $\bar{T}(x, y, z)$ and $\bar{\rho}(x, y, z)$ are the temporally averaged atmospheric temperature and density, respectively.

\section{The atmospheric MEP model}

Our MEP model is illustrated in Fig. 2. It has a series of boxes, each representing the zonally and vertically averaged atmosphere over a chosen latitude range. We will use 64 boxes, yielding the same latitudinal resolution as in the CAM3 simulation. Each box is warmed by solar radiation $F_{S}$ and convective heat flux $F_{\mathrm{CHF}}$ from the surface. The latter comprises latent and sensible heat fluxes, defined as positive if the atmosphere gains heat. The surface radiates as a blackbody while the atmosphere radiates as a graybody, equally in both directions with an emissivity $\epsilon$. A fraction $\epsilon$ of the terrestrial radiation is absorbed by the atmosphere, while the remaining is emitted to space. The surface temperatures $T_{S}$ are fixed and prescribed, in keeping with the AGCM. The only energy transport between the boxes is the meridional heat flux $F_{H}$.

Assuming energy balance in each box yields

$$
F_{S, i} A+\epsilon \sigma T_{S, i}^{4}-2 \epsilon \sigma T_{i}^{4}+F_{\mathrm{CHF}, i}+\Delta F_{H, i}=0 \quad(\text { box } i),
$$

where $F_{S, i}=I_{S, i}(1-R)$, where $I_{S, i}$ is the incoming solar radiation at the TOA in box $i, R$ is the albedo, $A$ is the atmospheric absorptivity, $\sigma$ is the Stefan-Boltzmann constant, and $\Delta F_{H, i}=F_{H, i-1}-F_{H, i}$ is the meridional heat convergence. There are thus three unknowns in the equation: $T_{i}, F_{\mathrm{CHF}, i}$, and $\Delta F_{H, i}$. 


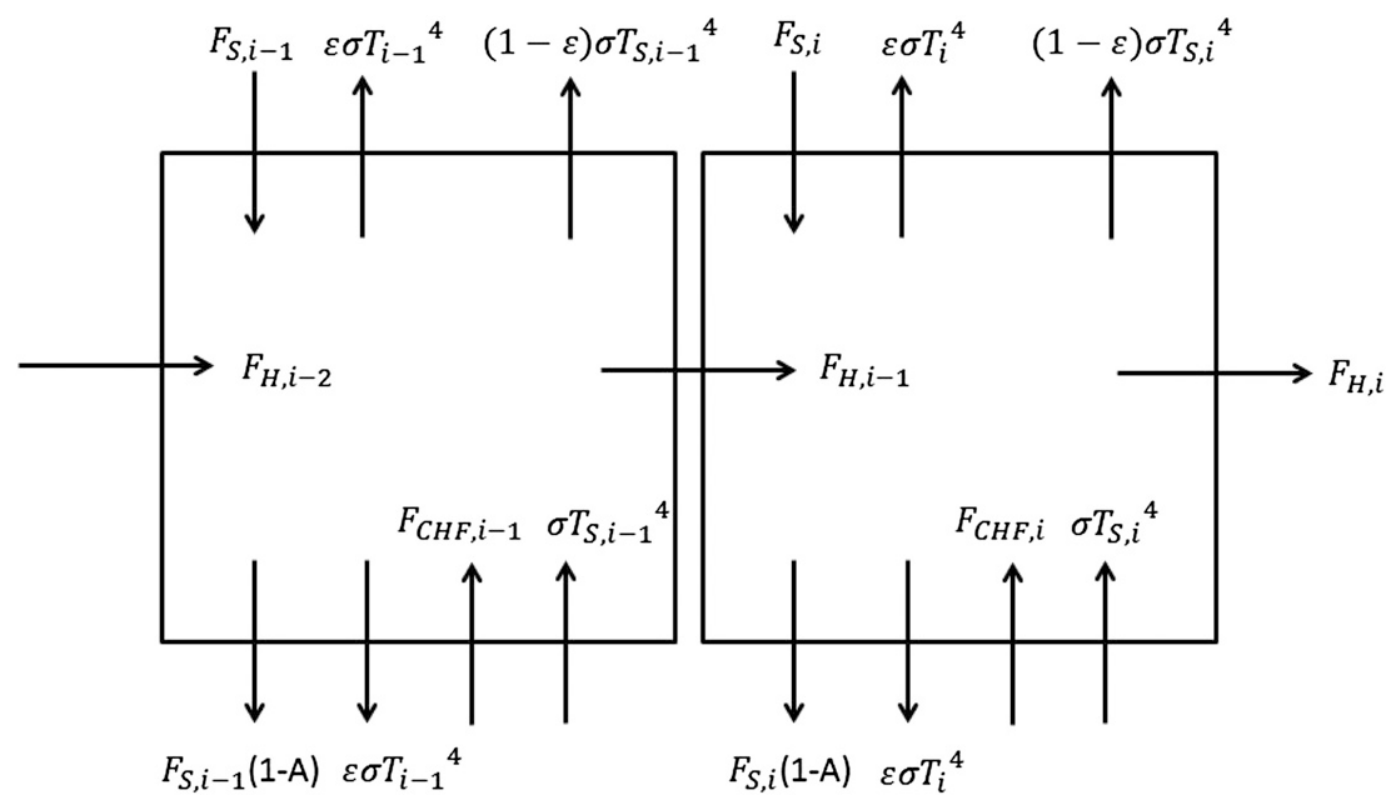

FIG. 2. The atmospheric EBM (AMEP) used in the present study. Each box represents an atmospheric column in equilibrium.

MEP yields a second equation, as discussed below, but we require one more. So we make a further assumption that the convective heat fluxes serve to maximize entropy production in the vertical. We impose this constraint first, and then use MEP to determine the lateral heat convergence $\Delta F_{H}$.

Finding the two fluxes separately is a standard procedure (Paltridge 1975, 1978; O'Brien and Stephens 1995). ${ }^{2}$ Results from Pascale et al. (2012) support the separation of the entropy production. In fact, both convective and lateral fluxes can be maximized simultaneously, but the calculation is somewhat simpler when done separately.

\section{a. The MEP convective heat flux}

To find the convective fluxes, we use an EBM with two vertical boxes, one representing the atmosphere and the other the surface (Fig. 3). Ozawa and Ohmura (1997) also invoked MEP to study convective heating, with continuous variations in the vertical. A two-box model suffices here, as we seek to determine how convective heating depends on the temperature difference between the surface and atmosphere. The boxes are in energy balance separately, so that the

\footnotetext{
${ }^{2}$ Paltridge (1975, 1978) and O'Brien and Stephens (1995) make the same assumption, although they maximized vertical convective heating rather than entropy production. They also had active surface layers (i.e., with lateral "ocean" heat fluxes).
}

combined system is also in radiative balance (the incoming solar radiation equals the outgoing radiation at TOA). The energy balance equations for the atmosphere and the surface are

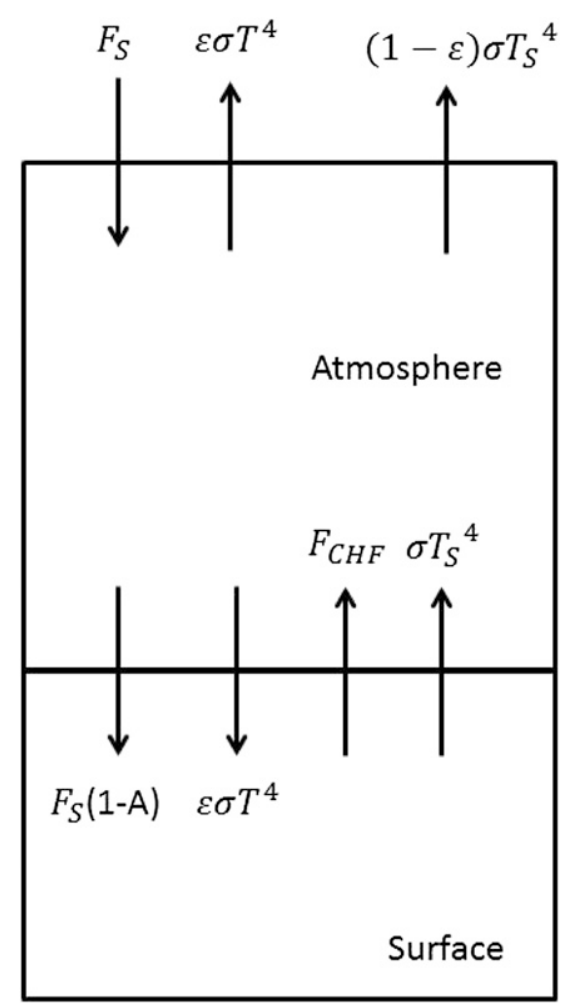

FIG. 3. A two-box EBM representing the atmosphere and the surface layer. 

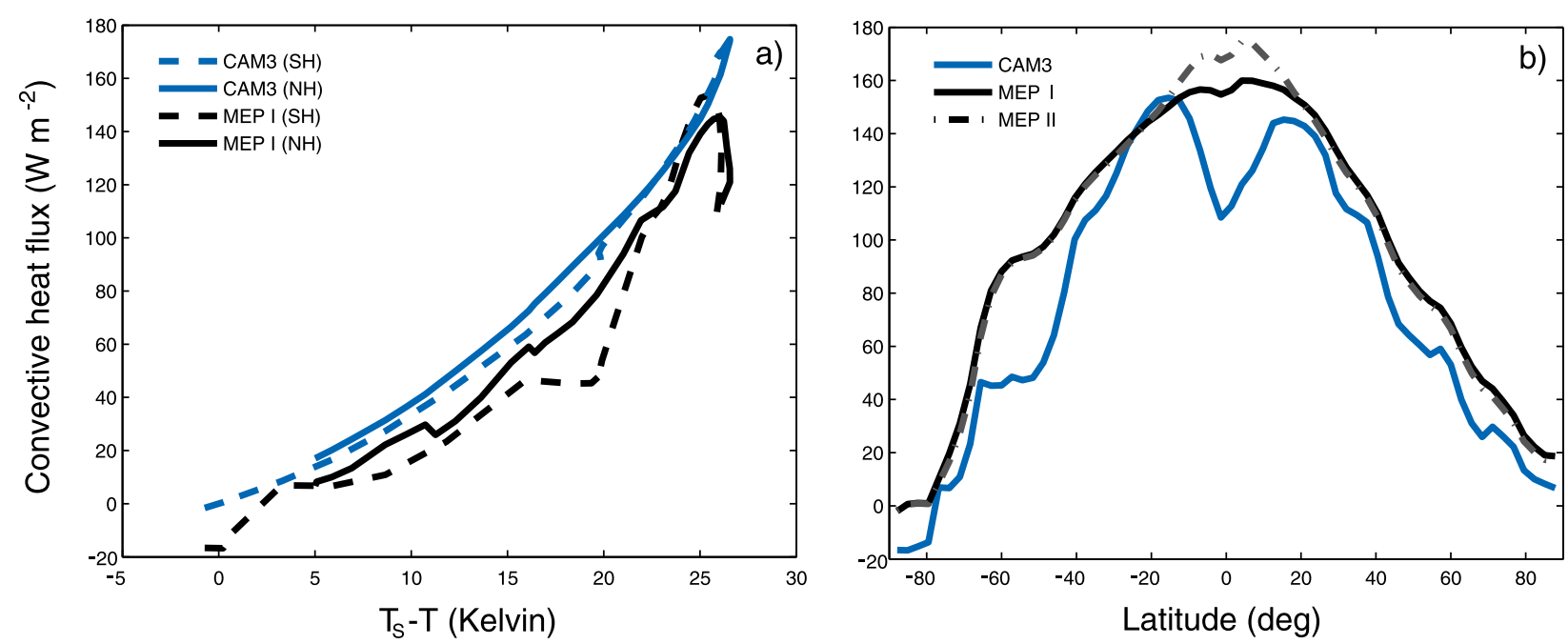

FIG. 4. (a) The convective heat fluxes in the CAM3 control run [blue curves, dashed (SH) and solid (NH)] plotted against the difference between the mean atmospheric temperature and the surface temperature, with the MEP prediction [see (9)] with global-mean emissivity for comparison (black curves). (b) The convective fluxes as a function of latitude from CAM3 (blue line), the MEP estimate with constant emissivity (black solid line), and the MEP estimate with zonally varying emissivity (black dash-dotted line).

$F_{S} A+F_{\mathrm{CHF}}+\epsilon \sigma T_{S}^{4}-2 \epsilon \sigma T^{4}=0 \quad$ (Atmosphere)

and

$F_{S}(1-A)+\epsilon \sigma T^{4}-F_{\mathrm{CHF}}-\sigma T_{S}^{4}=0 \quad$ (Surface).

The TOA balance is their sum:

$$
F_{S}-\epsilon \sigma T^{4}-(1-\epsilon) \sigma T_{S}^{4}=0
$$

The unknowns here are $T, T_{S}$, and $F_{\mathrm{CHF}}$. We close the system by maximizing entropy production, using the following cost function:

$$
L=F_{\mathrm{CHF}}\left(\frac{1}{T}-\frac{1}{T_{S}}\right)+\beta\left[F_{S}-\epsilon \sigma T^{4}-(1-\epsilon) \sigma T_{S}^{4}\right] .
$$

The first portion of $L$ is the entropy production associated with the convective flux while the second ensures the total radiation balance is closed, with $\beta$ as a Lagrange multiplier. We maximize $L$ by taking the derivative with respect to both $T$ and $T_{S}$, yielding two equations. Eliminating $\beta$ between them yields

$$
F_{\mathrm{CHF}}=\frac{4 \sigma \epsilon(2-\epsilon) T^{4} T_{S}^{4}\left(T_{S}-T\right)}{(1-\epsilon) T_{S}^{5}+\epsilon T^{5}} .
$$

Of course, the system can be solved completely to determine $T$ and $T_{S}$, but we seek only a relation between $F_{\mathrm{CHF}}$ and the temperatures. The flux is a nonlinear function of the temperatures and also has a nontrivial dependence on the emissivity.

How realistic is the estimate? The prediction is compared with the observed convective (latent plus sensible) fluxes from the CAM3 control run (section 5a) in Fig. 4. The CAM3 fluxes are plotted against $T_{S}-T$, where $T$ is the density-weighted temperature in the atmosphere (section 5a), averaged zonally and also over the 20 -yr period of the simulation. The prediction (9) is indicated by the black curves, while the result from CAM3 is shown by the blue curves. Note that (9) has only a single parameter, the emissivity. For this, we use the same value as in the control run. The details on how this was determined are given in section 5a. For the comparison Fig. 4a, we use the deduced global-mean value, $\epsilon=0.84$. The resulting fluxes from MEP are somewhat larger than predicted, particularly in the Southern Hemisphere ( $\mathrm{SH})$, but the comparison is nevertheless surprisingly good.

If one uses (9) to predict the convective fluxes as a function of latitude, one obtains the results shown in Fig. 4b. As in CAM3, the predicted fluxes are largest in the tropics and decrease toward the poles, in both hemispheres. The MEP flux also captures the asymmetry between the two hemispheres, with lower fluxes in the SH. However, the MEP estimate fails to reproduce the pronounced minimum at the equator seen in CAM3, where the fluxes are roughly $20 \%$ less than at $20^{\circ} \mathrm{N}$ and $20^{\circ} \mathrm{S}$. The MEP fluxes in contrast are largest at the equator. The fluxes in CAM3 depend on the mean (Hadley) circulation and are evidently not well captured by MEP. In addition, the occurrence of high-level clouds 
due to deep convection in the intertropical convergence zone (ITCZ) can reduce the atmospheric emissivity and are not taken properly into account by the prescribed distribution of emissivity. Nevertheless, the MEP expression in (9) appears to be sufficiently good for studying midlatitude heat fluxes.

In fact, the emissivity is not constant but varies with latitude in CAM3. Using this in the MEP estimate yields the dash-dotted curve in the figure. The fluxes are essentially identical and the mid- and high latitudes but are also somewhat larger at the equator. So using this only exacerbates the discrepancy from CAM3 there.

The rate of entropy production due to convective heating for the two MEP solutions are 35.5 and $36.2 \mathrm{~mW} \mathrm{~m}^{-2} \mathrm{~K}^{-1}$ for the constant emissivity and the zonally varying emissivity cases, respectively. This is in agreement with the results from Pascale et al. (2011), who found the entropy production due to convective heating to be $38 \mathrm{~mW} \mathrm{~m}^{-2} \mathrm{~K}^{-1}$ in a GCM.

\section{b. The horizontal heat fluxes}

With the convective heat parameterization, we can write the energy balance for the atmospheric box as

$$
\begin{aligned}
& F_{S, i} A+\epsilon \sigma T_{S, i}^{4}-2 \epsilon \sigma T_{i}^{4}+\frac{4 \sigma \epsilon(2-\epsilon) T_{i}^{4} T_{S, i}^{4}\left(T_{S, i}-T_{i}\right)}{(1-\epsilon) T_{S, i}^{5}+\epsilon T_{i}^{5}} \\
& +\Delta F_{H, i}=0 \quad(\operatorname{box} i) .
\end{aligned}
$$

Only $T_{i}$ and $\Delta F_{H, i}$ are unknown. Following O'Brien and Stephens (1995), we introduce a second cost function, related to the entropy production from the meridional heat transport.

Heat is only redistributed by the meridional fluxes in the model, so their sum must vanish:

$$
\sum_{i=1}^{64} \Delta F_{H, i} \cos \left(\phi_{i}\right)=0 .
$$

The $\cos (\phi)$ factor accounts for the different surface areas of the boxes, with larger areas at low latitudes. The cost function is then written as

$$
L=\sum_{i=1}^{64}\left(\frac{\Delta F_{H, i}}{T_{i}}+\beta \Delta F_{H, i}\right) \cos \left(\phi_{i}\right) .
$$

As with (8), the first term is the entropy production, and the second enforces conservation of the total heat. Maximizing $L$ with respect to $T_{i}$ yields

$$
T_{i} \frac{\partial \Delta F_{H, i}}{\partial T_{i}}\left(1+\beta T_{i}\right)-\Delta F_{H, i}=0 .
$$

For $\Delta F_{H, i}$ and its derivative, we use the energy balance in (10). The resulting equation is a strongly nonlinear function of $T_{i}$. One can demonstrate that it has a single solution in the range of relevant parameters. We solve it numerically, using a specific value of $\beta$; we then adjust $\beta$ and repeat the process until the total heat transport [see (11)] balances (Paltridge 1975; O'Brien and Stephens 1995). The solution yields the atmospheric temperature in the boxes as well as the meridional heat fluxes.

\section{Results}

\section{a. Control run}

We first compare the AMEP solution to the control run with the AGCM. For this, we must specify the three radiation parameters (planetary albedo, atmospheric absorptivity, and atmospheric emissivity) and the surface temperatures in AMEP. The shortwave parameters are calculated using a single-layer solar radiation model and data from CAM3, following the approach of Donohoe and Battisti (2011). Emissivity values of 1 are easily reached under cloudy conditions, whereas with clear skies the emissivity can have a range of values and depends on temperature, humidity, clouds, and stratification. The atmospheric emissivity was also calculated using a single-layer radiation model and data from CAM3. As such, the calculated emissivity is affected by humidity, clouds, and stratification, factors otherwise excluded in the MEP calculation. We also ran AMEP using an emissivity formulation modified by a cloudiness correction (Swinbank 1963; Crawford and Duchon 1999), but the results were similar to those obtained without this.

The parameters deduced from CAM3 are shown in Fig. 5. The absorptivity $A$ is nearly constant over the range of latitudes, with a value near 0.25 . The other two parameters vary with latitude. The albedo is largest at high latitudes, decreasing to a value of roughly 0.25 in the tropics. The emissivity has the opposite behavior, being small at high latitudes and increasing to values near 1 at low latitudes.

AMEP was run using these zonally varying parameters and also using constant parameters. The latter were the global means: an albedo of $R=0.33$, an absorptivity of $A=0.23$, and an emissivity of $\epsilon=0.84$. The results for both are shown in Fig. 6, with the latitudinally varying parameters indicated by the dash-dotted curves and the global-mean values indicated by the solid curves. The figure compares the AMEP temperature, convective heat flux, and meridional heat transport with the corresponding means from CAM3. Using the latitudinally varying parameters evidently has only a weak effect on the results. As noted before, the largest difference is with the convective fluxes near the equator, which are 


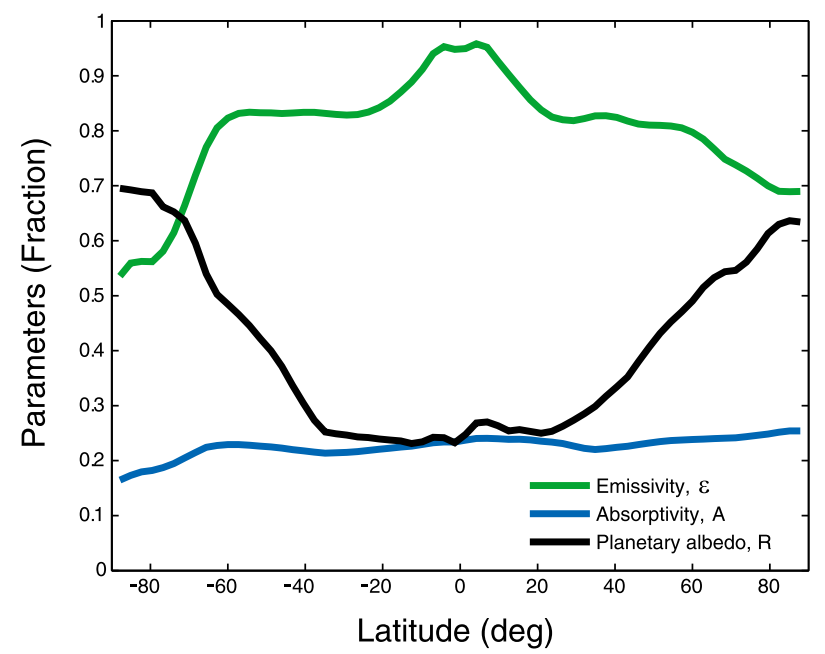

FIG. 5. Temporally and zonally averaged atmospheric emissivity (green line), absorptivity (blue line), and planetary albedo (black line).

slightly larger. Using the varying parameters also has little effect on the difference fields for the perturbed runs, so we use the global mean parameter values in the subsequent calculations, for simplicity.

In comparing the AMEP temperature with the density-weighted average in CAM3, we found that the AMEP values were, on average, larger (Fig. 6a). This is probably not surprising since AMEP has only a single temperature in the vertical. In comparing the two temperature profiles, we also subtract $10 \mathrm{~K}$ from the AMEP temperature distribution for visualization (Fig. 6a, green line). The AMEP and CAM3 temperature fields are similar. Both exhibit maxima in the tropics with weak gradients and then decrease monotonically toward the poles. Both distributions are also asymmetric, being warmer in the Northern Hemisphere (NH) than in the SH. However, the AMEP temperatures have weaker meridional gradients. This is due to more efficient heat transport in AMEP, as seen below. Furthermore, AMEP is warmer over Antarctica. Here CAM3 has an ice sheet and thus dynamics that are not captured by the simplified EBM.

The convective heat fluxes (Fig. 6b) are also similar. Note that this figure is different from Fig. 4b, which simply showed the MEP convective parameterization applied to the control run temperatures from CAM3; this is the convective flux for the full AMEP solution. The fields are essentially identical in the $\mathrm{NH}$ and are very similar in the SH. However, as in Fig. 4b, the AMEP convective fluxes are too large in the tropics and fail to reproduce the minimum at the equator seen in CAM3.

The northward heat transport is shown in Fig. 6c. Here the differences are greater. While AMEP is qualitatively
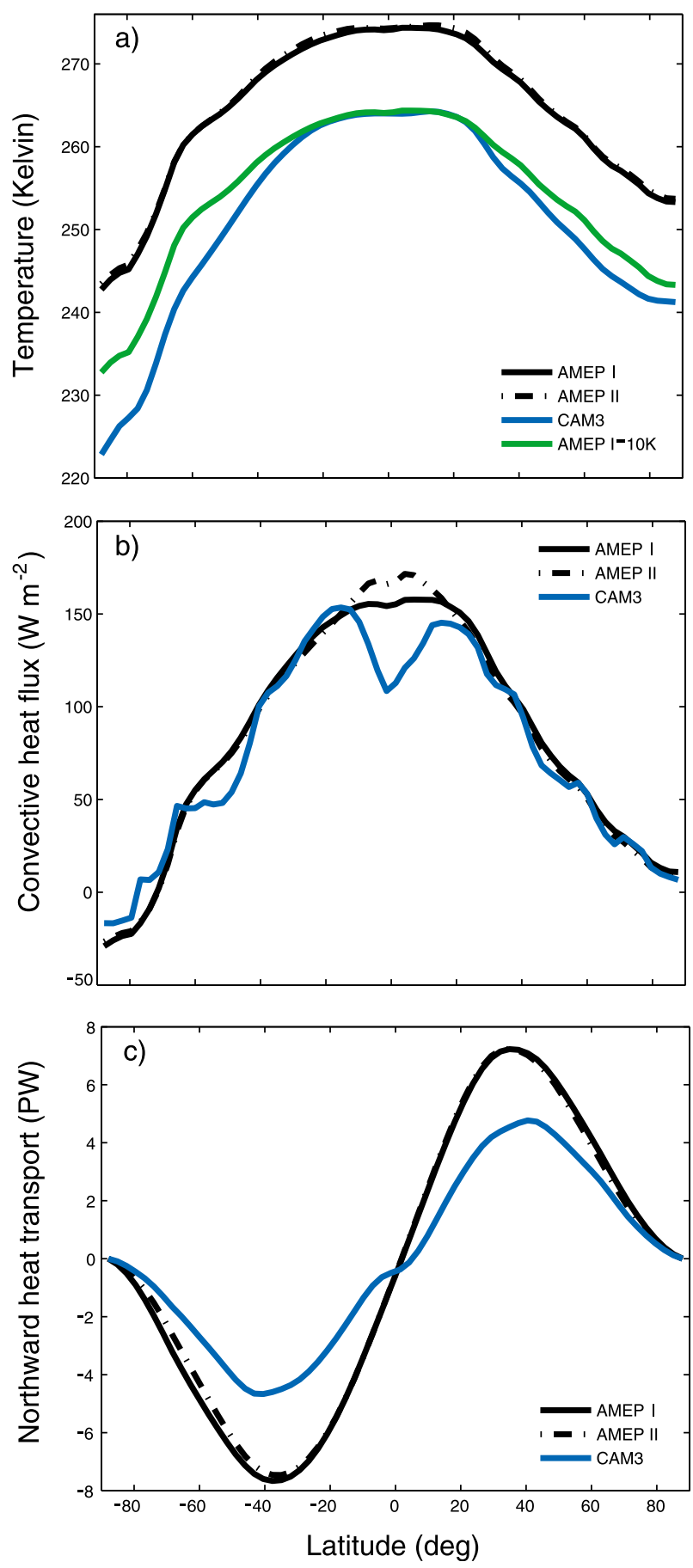

FIG. 6. AMEP solution with constant parameter values (black solid line), AMEP solution with zonally varying parameter values (black dash-dotted line), and CAM3 (blue line) control run results: (a) mean atmospheric temperature, (b) convective heat flux, and (c) northward heat transport. Also included in (a) is a green line for which $10 \mathrm{~K}$ has been subtracted from the AMEP solution for visualization. 
correct, having poleward fluxes in both hemispheres that are peaked at midlatitudes, the magnitudes are significantly larger than in CAM3. The AMEP maxima are 7.7 and 7.2 $\mathrm{PW}$ in the $\mathrm{SH}$ and the $\mathrm{NH}$, respectively, while the corresponding values for CAM3 are 4.7 and $4.8 \mathrm{PW}$. The greater transport in AMEP accounts for the weaker meridional temperature gradients seen in Fig. 6 a.

As noted, the maxima in heat transport are our proxy for the position of the storm tracks. These lay slightly equatorward in AMEP than in CAM3. A further, though less marked, difference occurs in the tropics. While the CAM3 transport flattens equatorward of $10^{\circ}$, it does not in AMEP.

There are several possible reasons for the difference in the magnitude of the heat transport. First, the local minimum in the convective heat flux at the equator is not present in AMEP. As such, the amount of heat supplied to the atmosphere in the tropics is $40 \mathrm{~W} \mathrm{~m}^{-2}$ greater in AMEP. This also accounts for the steeper increase in transport in the tropics in the latter. Second, the radiative cooling in the tropics is less in AMEP than in CAM3 (not shown), so there is more heat in the tropics that must be transported to higher latitudes.

A third possibility, though, was raised by one of the reviewers. Imagine that CAM3 had an oceanic slab. Then there would be a proxy oceanic heat transport, and this would increase the overall heat transport to the poles. This raises the question about whether there is a difference in the radiation budget at the surface in the models. The surface balance follows from that given in (5):

$$
\sum_{i} F_{S, i}(1-A)+\epsilon \sigma T_{i}^{4}-F_{\mathrm{CHF}, i}-\sigma T_{S, i}^{4} .
$$

Evaluating this for AMEP, we obtain a deficit of $-69 \mathrm{~W} \mathrm{~m}^{-2}$, while for the control run with CAM3, the result is $-100 \mathrm{~W} \mathrm{~m}^{-2}$. That both values are fairly large reflects that (14) is too simple to fully capture the radiation balance at the surface. That the AMEP and CAM3 values differ is related to the vertical variation of temperature. The atmospheric temperature "felt" by the surface is that of the layer over the surface, and this is significantly warmer than the density-weighted average. We find, accordingly, that the surface budget in CAM3 is approximately balanced if $26.5 \mathrm{~K}$ is added to $T_{i}$ in the surface budget. This corresponds to a temperature which is $5.5 \mathrm{~K}$ cooler than the surface temperature. Similarly, if one adds $17 \mathrm{~K}$ to the AMEP temperature, the surface budget also balances; this is nearly the same as the CAM3 correction, if the $10-\mathrm{K}$ mean offset is taken into account. Therefore, although the deficits evidently differ in the two models, there does not appear to be a systematic difference in the surface budgets.
Despite these discrepancies, AMEP provides a reasonable first-order description of the CAM3 fields. Furthermore, the entropy production rates in the AMEP solutions, 31.6 and $17.9 \mathrm{~mW} \mathrm{~m}^{-2} \mathrm{~K}^{-1}$ for the convective and meridional heat transports, respectively, agree well with the material entropy production rate found previously by Pascale et al. (2011). The success of AMEP suggests that the transport in CAM 3 is largely a matter of redistributing heat and that the transport is close to what it would be if entropy production were maximized. The results thus far are in line with Paltridge $(1975,1978)$ and with other studies. However, the question remains as to what extent AMEP can simulate changes in atmospheric fields due to perturbed surface temperatures.

\section{b. Aquaplanet solutions}

As noted, the SSTs are increased and decreased in the sensitivity experiments. However, the actual forcing on the atmosphere differs because CAM3 also has land, where the temperatures are allowed to adjust. But before we study the response to the full surface temperature fields, it is useful to see how AMEP responds to the imposed SST changes alone. Doing this is equivalent to studying the response in an aquaplanet simulation (e.g., Bengtsson et al. 2006; Brayshaw et al. 2008; Lu et al. 2010).

We consider the surface forcings shown in Fig. 7a. These are simple 2-K-warming or -cooling profiles, corresponding to the imposed heating or cooling shown in Fig. 1. Note the temperature perturbations return to zero over a single grid point, yielding strong gradients at the edges of the regions; this contrasts with the $8^{\circ}$ transition range used in the CAM3 simulations (section 3).

The resulting AMEP temperature profiles are shown in Fig. 7b. Shown are the differences in air temperature from the control (unperturbed) solution shown in Fig. 6a. The temperature differences largely mirror the forcing, including the strong gradients on the edges. Of course AMEP has no thermal wind shear or baroclinic instability, so such gradients are possible. The $2 \mathrm{~K}$-lowlat solution has warmer temperatures at low latitudes $\left(45^{\circ} \mathrm{S}-\right.$ $45^{\circ} \mathrm{N}$ ), having warmed by $1.6 \mathrm{~K}$, somewhat less than the 2-K difference imposed, but the temperatures are also warmer at high latitudes by $0.7 \mathrm{~K}$, so heat is obviously being transported to these latitudes.

The warming in the $2 \mathrm{~K}$ solution is uniform, in line with the forcing, but is also less than the forcing, about $1.75 \mathrm{~K}$. The magnitude of the warming would seem to be predictable, given a constant increase in SST, but the calculation is not trivial given the nonlinear dependence on temperature of the convective flux. In the $2 \mathrm{~K}$-tropics solution there is a $1.2-\mathrm{K}$ warming in the tropics and a $0.3-\mathrm{K}$ warming elsewhere. In the other cases, the temperature response also resembles the forcing, with a smaller 

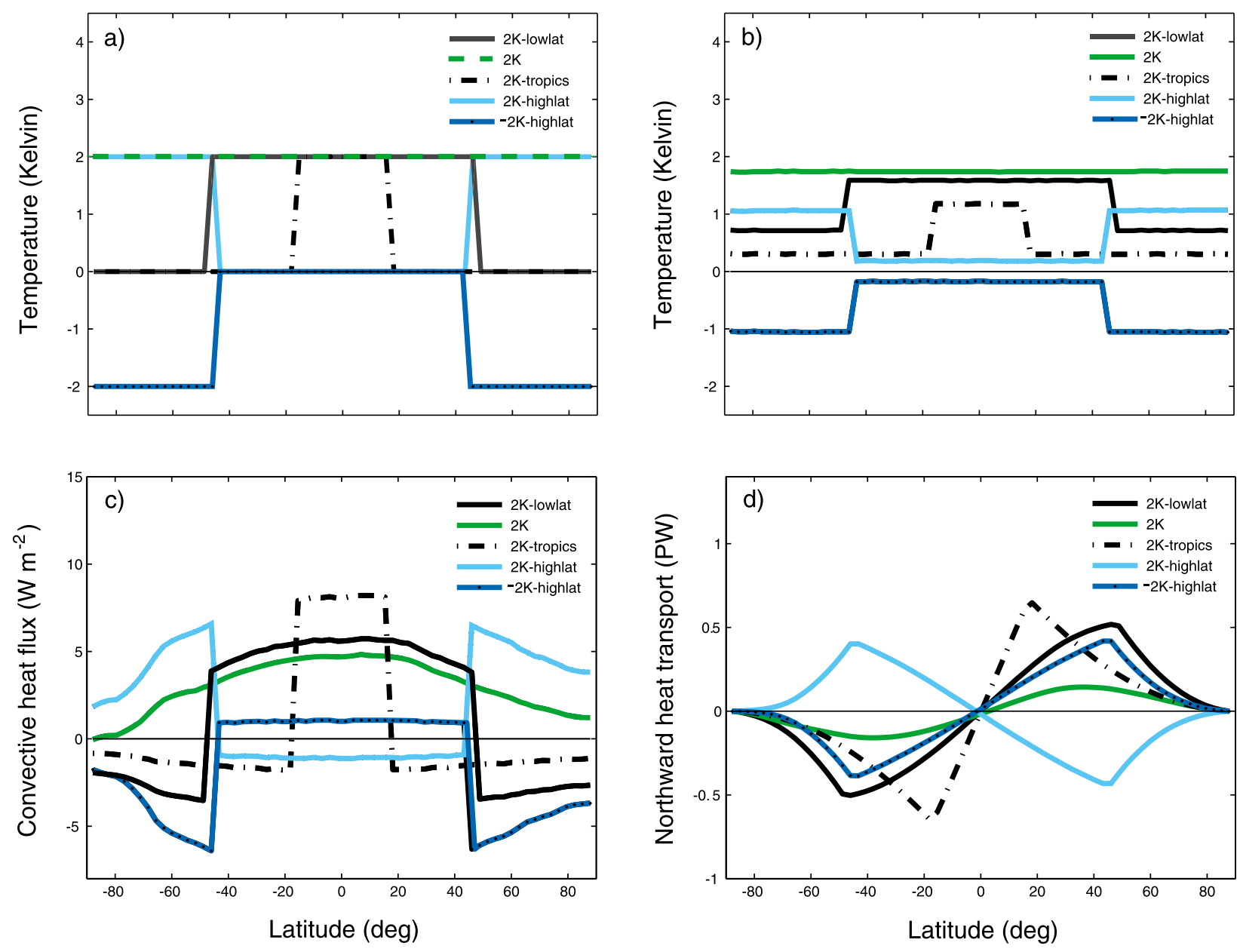

FIG. 7. Aquaplanet solutions from AMEP: (a) SST forcing and difference plots of (b) mean atmospheric temperature, (c) convective heat flux, and (d) northward heat transport. The different lines correspond to the SST sensitivity runs as indicated in the legends.

amplitude. The temperature is always affected in the unforced latitudes as well, including in the $\pm 2 \mathrm{~K}$-highlat solutions.

The differences in convective heating are shown in Fig. 7c. Here, too, the response largely resembles the forcing. The largest change occurs in the $2 \mathrm{~K}$-tropics run, with an increase of $8 \mathrm{~W} \mathrm{~m}^{-2}$ at low latitudes. The increase in the $2 \mathrm{~K}$-lowlat, on the other hand, is only about $5 \mathrm{~W} \mathrm{~m}^{-2}$. This difference is consistent with the convective heat flux's dependence on the air-sea temperature difference in (9). In the $2 \mathrm{~K}$-lowlat solutions, the air warms more than in the $2 \mathrm{~K}$-tropics solution, so the airsea temperature difference is less. The increase in convective heating is less still in the $2 \mathrm{~K}$ solution. Note that though that the difference decreases with latitude, despite that, both the air and sea temperatures have been increased uniformly. This stems from the convective flux being not just proportional to the temperature difference but also to the temperatures themselves. Notice also that the changes for the $2 \mathrm{~K}$-lowlat and the $-2 \mathrm{~K}$ highlat solutions are very similar, albeit with a change in sign.

Finally, there are the changes in the meridional heat transport (Fig. 7d; to be compared with the total heat transport shown in Fig. 6). The maximum transport is about $7.5 \mathrm{PW}$, so the changes here are on the order of $5 \%-10 \%$. In the $2 \mathrm{~K}$-lowlat solution, the difference curve peaks at $46^{\circ}$, poleward of the peak in the control at $38^{\circ}$. The peak in the $2 \mathrm{~K}$-tropics solution is instead at $18^{\circ}$, equatorward of that in the control. The changes in the $2 \mathrm{~K}$ solution, on the other hand, are broad and peaked at the same location as in the control solution. The peak in the $-2 \mathrm{~K}$-highlat case is close to that in the $2 \mathrm{~K}$-lowlat solution.

Many of these changes are in line with the results of GL12. However, the changes here are also single signed in each hemisphere. With a shifting storm track, on the other hand, the difference field is typically dipolar; 
a poleward shift, for instance, has a negative difference equatorward and a positive one poleward. When the change is single signed and on the order of $5 \%-10 \%$, it may not produce a noticeable shift in the position of the maximum transport. This will be seen to be the case later on.

There are other points of interest. One is that the maximum change in heat transport occurs where the convective heat flux difference changes sign. For instance, in the $2 \mathrm{~K}$-tropics run, the additional heating occurs equatorward of $18^{\circ}$, and the peak transport difference is at $18^{\circ}$. Thus, convective heating plays a central role in determining the heat transport changes in AMEP. This makes sense as the additional heat introduced from the surface must be transported poleward. Second, the changes in the $2 \mathrm{~K}$-lowlat and $-2 \mathrm{~K}$-highlat runs are remarkably similar, so having excess heating at the low latitudes is essentially equivalent to having a heating deficit at high latitudes. This is also consistent with heat simply being redistributed in AMEP.

Of course, we do not know how realistic these solutions are, since we have not conducted aquaplanet simulations. The solutions are nevertheless enlightening for the more complicated CAM3 solutions, which are discussed next.

\section{c. Full perturbation solutions}

As noted, the SST perturbations shown in Fig. 1 represent only part of the surface heating in CAM3, because the land can warm. Figure 8 shows the difference in the total surface temperatures between the sensitivity runs and the control run for CAM3. Consider the 2K-lowlat run (Fig. 8a, black line). The temperature perturbation is approximately $2 \mathrm{~K}$ at low latitudes, but it is also nonzero at higher latitudes. In the SH, the difference is small at midlatitudes but increases over Antarctica, while in the $\mathrm{NH}$ there is warming over the entire range of latitudes and the difference does not fall below $1 \mathrm{~K}$. The asymmetry between hemispheres occurs because there is a larger land fraction in the $\mathrm{NH}$.

The other low-latitude warming runs are similar. In the $2 \mathrm{~K}$ run (green line), the surface temperatures are increased by $2 \mathrm{~K}$ over much of the globe, but there is a minimum over the Southern Ocean. In the 2K-tropics run (dash-dotted line), the surface temperature is $2 \mathrm{~K}$ warmer equatorward of $15^{\circ}$ but is also warmer at high latitudes. In the 2K-highlat run (Fig. 8b, light blue line), the surface temperatures warm somewhat less than the $2 \mathrm{~K}$ imposed on the SSTs, as the land does not warm as much. Also, the warming in the $\mathrm{SH}$ is greater than in the $\mathrm{NH}$, as there is more ocean. The differences in the $\pm 2 \mathrm{~K}$-highlat runs likewise differ from the changes imposed on the SST.

To compare with the perturbation experiments in CAM3, we use these averaged surface temperatures to
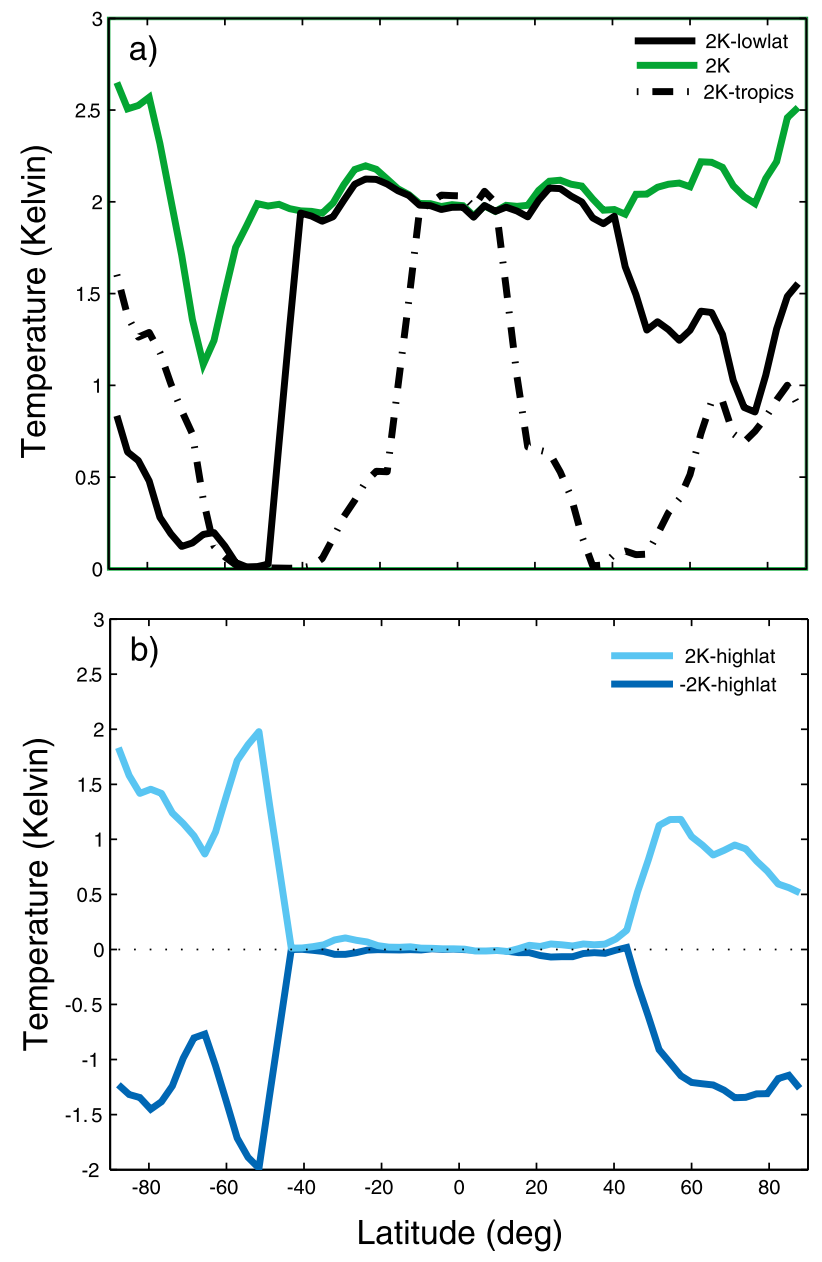

FIG. 8. Temporally and zonally averaged surface temperatures from the sensitivity runs minus the control run in CAM3: (a) $2 \mathrm{~K}$ lowlat (black solid line), $2 \mathrm{~K}$ (green line), and 2K-tropics (black dash-dotted line) runs; and (b) 2K-highlat (light blue line) and $-2 \mathrm{~K}$-highlat (dark blue line) runs. The temperatures are used as a boundary forcing in AMEP.

perturb $T_{S}$ in AMEP. The resulting difference fields for the atmospheric temperature are shown in Figs. 9a and 9c, with the corresponding CAM3 results in Figs. 9b and 9d. We separate the low-latitude cases (2K-lowlat, $2 \mathrm{~K}$, $2 \mathrm{~K}$-tropics) and the high-latitude cases $( \pm 2 \mathrm{~K}$-highlat) for clarity.

The AMEP temperature differences, as in the aquaplanet solutions, resemble the forcing but have a smaller amplitude. In the $2 \mathrm{~K}$-lowlat solution, the temperatures are $1.6 \mathrm{~K}$ warmer equatorward of $45^{\circ}$ and are also warmer at higher latitudes, mirroring the asymmetry in the forcing (Fig. 8). In contrast, the atmospheric temperatures are greater than the forcing in CAM3, increasing by $2.6 \mathrm{~K}$ in the tropics. The difference moreover extends past $45^{\circ}$, so that the warming extends over a larger range of latitudes. 

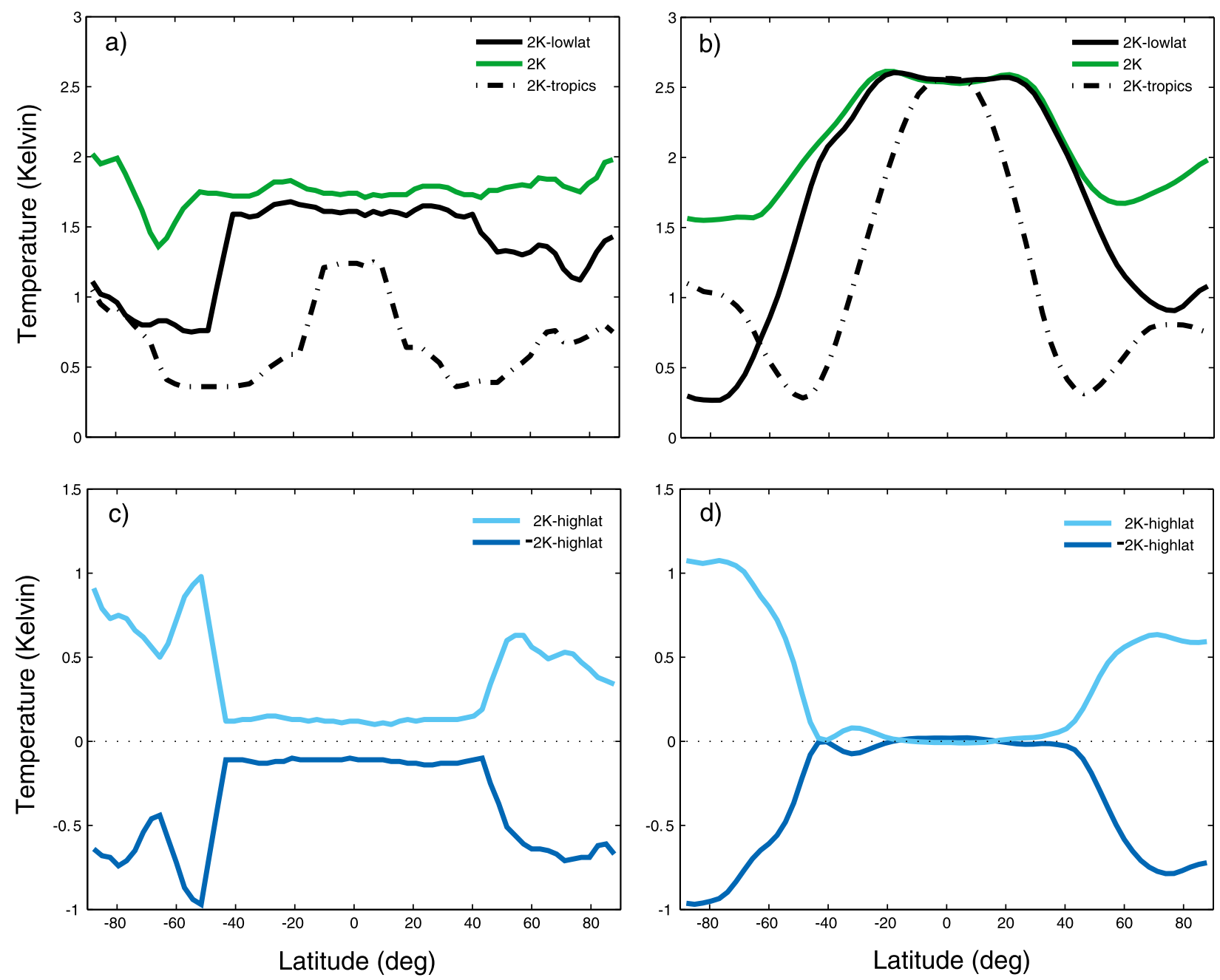

FIG. 9. Zonally averaged difference plots of the mean atmospheric temperature: (top) $2 \mathrm{~K}$-lowlat (black solid line), $2 \mathrm{~K}$ (green line), and 2K-tropics (black dash-dotted line) solutions from (a) AMEP and(b) CAM3; and (bottom) 2K-highlat (light blue line) and -2K-highlat (dark blue line) solutions from (c) AMEP; and (d) CAM3.

Similar comments apply to the other low-latitude cases. While the AMEP temperature differences resemble the forcing, the temperature increases in the CAM3 cases are similar at low latitudes. As such, the temperature increase in the $2 \mathrm{~K}$-tropics run is fully 2.5 times greater than in the AMEP solution. The temperature difference in the $2 \mathrm{~K}$ run moreover is qualitatively similar to that in the $2 \mathrm{~K}$-lowlat run-in contrast with the AMEP $2 \mathrm{~K}$ solution, where the temperature differences are nearly constant with latitude.

The high-latitude cases, on the other hand, are more similar. Both AMEP and CAM3 produce temperature differences of roughly $0.5 \mathrm{~K}$ at high latitudes in the $\mathrm{NH}$ and increasing to $1 \mathrm{~K}$ in the $\mathrm{SH}$. The response in both models resembles the surface forcing, albeit with a smaller amplitude. AMEP exhibits a small increase or decrease in temperature across the low latitudes as well, which is absent in CAM3, but this is fairly small (on the order of $0.1 \mathrm{~K}$ ).

The convective heat flux differences are shown in Fig. 10. Despite the excellent agreement in the control run comparison, AMEP underestimates the changes in the perturbation experiments. In the $2 \mathrm{~K}$-lowlat run (Fig. 10a), the change in the AMEP fluxes resembles the surface forcing, with an amplitude of approximately $6 \mathrm{~W} \mathrm{~m}^{-2}$. This is similar to the result obtained with the aquaplanet solution. As there, the difference mirrors the forcing, producing strong gradients at the edges. The differences for CAM3 (Fig. 10b) are similar in shape but nearly twice as large. Thus, the gradients, at roughly the same latitudes as in AMEP, are much greater.

Similar comments apply for the $2 \mathrm{~K}$ case, with the exception that the steep gradients are not present at 

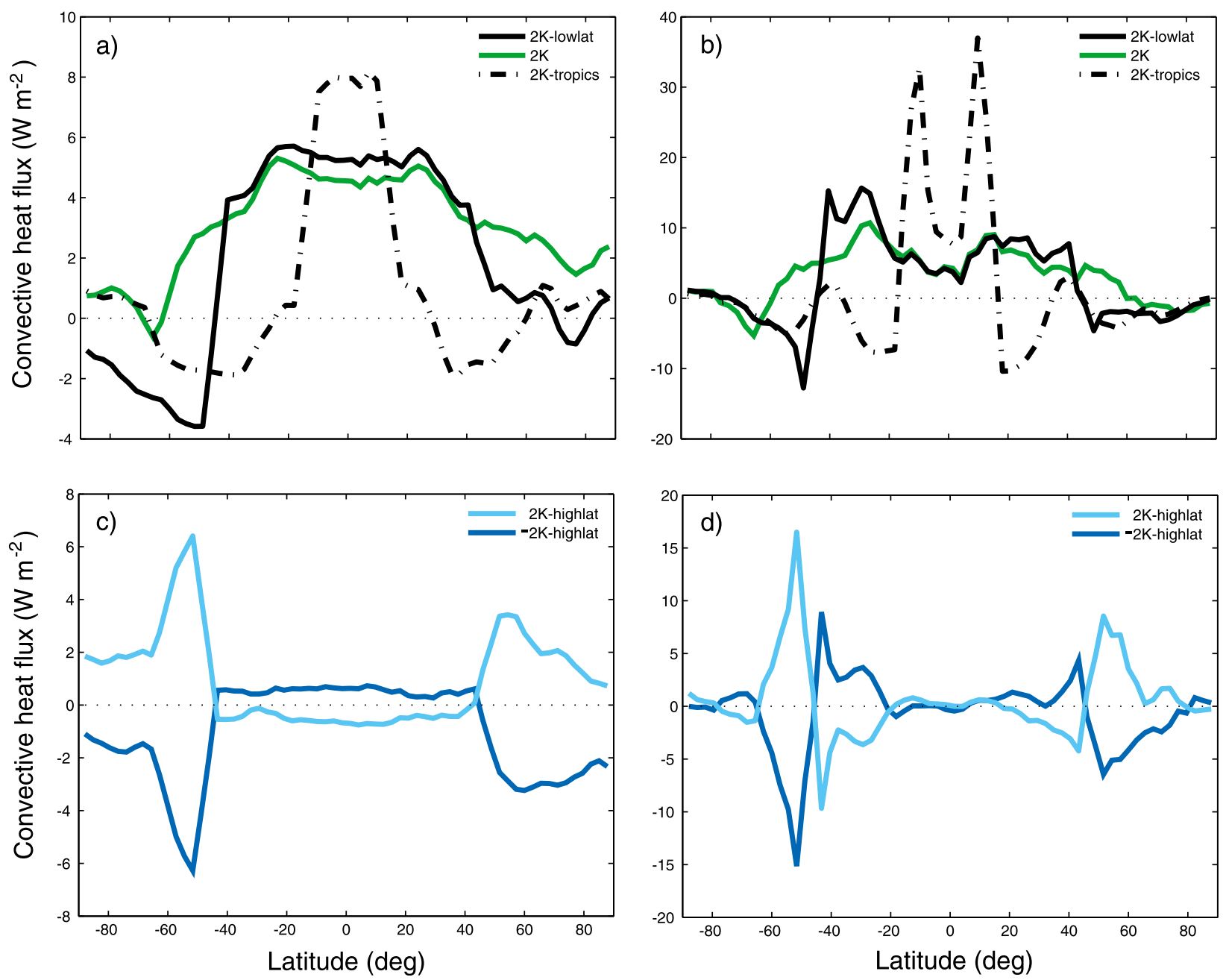

FIG. 10. Zonally averaged difference plots of the convective heat flux (i.e., the latent plus sensible heat flux): (top) 2K-lowlat (black solid line), 2K (green line), and 2K-tropics (black dash-dotted line) solutions from (a) AMEP and (b) CAM3; and (bottom) 2K-highlat (light blue line) and -2K-highlat (dark blue line) solutions from (c) AMEP and (d) CAM3.

midlatitudes. The $2 \mathrm{~K}$-tropics case shows the largest increases of all the runs, in both models. In both cases, there is an increase in the tropics, a decrease in the subtropics, and an increase again in higher latitudes. But the largest CAM3 differences are nearly 4 times larger than the largest AMEP differences. CAM3 also shows the characteristic equatorial minimum, absent in AMEP.

The changes in the $\pm 2 \mathrm{~K}$-highlat runs are qualitatively similar and resemble the surface forcing, but again the differences are up to 2.5 times larger in CAM3 than in AMEP. The flux differences in both models change sign in the low latitudes, but in AMEP this occurs uniformly over the range of unforced latitudes; in CAM3 the response is localized just equatorward of the forcing region. Thus, the convective flux differences have a more dipolar structure in CAM3.
The changes in meridional heat transport are shown in Fig. 11. The heat transport from the control cases are also shown to indicate the location and amplitudes of the maxima in those cases. The changes in AMEP are quite similar to those in the aquaplanet solutions. These have the same sign in each hemisphere and span the range of latitudes. In the $2 \mathrm{~K}$-lowlat and in the $\pm 2 \mathrm{~K}$-highlat solutions, the peaks occur poleward of that in the control, while in the tropics solution, the peaks are equatorward. The changes are typically on the order of $5 \%$.

In CAM3, the changes are more localized. This is particularly noticeable in the $2 \mathrm{~K}$-tropics run, where the change is sharply peaked at $15^{\circ}$ in both hemispheres. In addition, the changes in several runs exhibit a dipolar structure. This is particularly noticeable in the $\mathrm{SH}$, but also to a lesser extent in the $\mathrm{NH}$ in the $\pm 2 \mathrm{~K}$-highlat runs. The magnitudes of the changes are also greater than in 

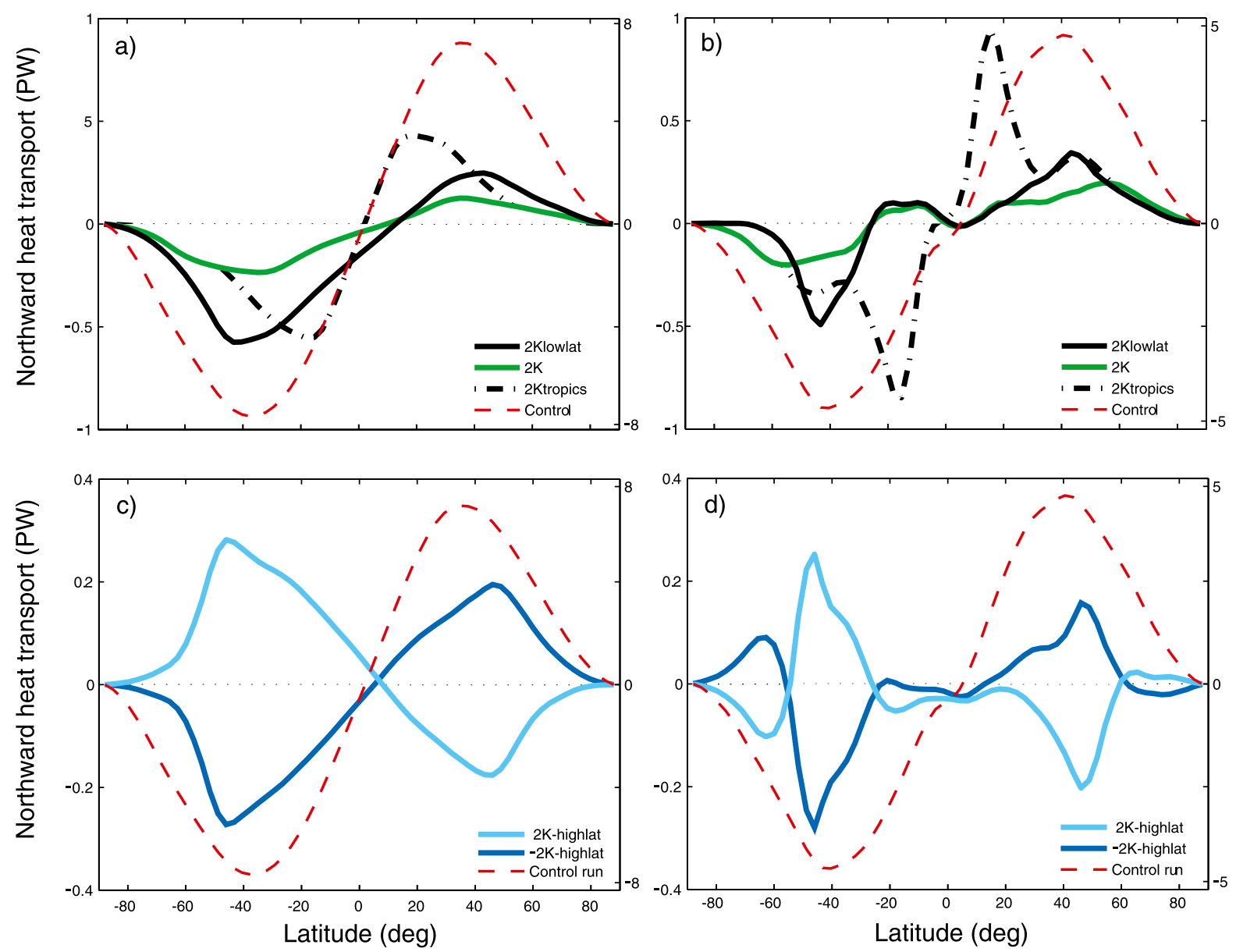

FIG. 11. Zonally averaged difference plots of the northward heat transport: (top) 2K-lowlat (black solid line), 2K (green line), and 2Ktropics (black dash-dotted line) solutions from (a) AMEP and (b) CAM3; and (bottom) 2K-highlat (light blue line) and -2K-highlat (dark blue line) solutions from (c) AMEP and (d) CAM3. Also included are the control solution/run (red dashed lines). Note the different $y$ axis.

AMEP. In the 2K-tropics run, the transport increases by roughly $20 \%$, as opposed to $7 \%$ in AMEP.

As noted in the aquaplanet solutions, the differences in transport do not necessarily imply a shift in the peak transport. Shown in Fig. 12 are the total heat transports in the control and 2K-lowlat solutions for both models, with insets showing close-ups in the regions of the peaks. In CAM3, the peaks do indeed shift poleward, in both hemispheres. These shifts are not large- on the order of $1^{\circ}$ (GL12) - but they are visible. No such shifts are evident in AMEP. Rather, the magnitude of the fluxes increases while maintaining their positions.

The differences can be linked directly to those in convective heating. As noted in the aquaplanet AMEP solutions, the peaks in transport difference occur where the difference in convective heating changes sign. In AMEP, an increase in convective heating in the forcing region is accompanied by a decrease outside, spread uniformly over the unforced latitudes. In CAM3, the corresponding decrease is more localized, occurring adjacent to the forced latitudes. Thus, the change in convective heating is greater at the crossover latitude, implying the transport difference is more localized. The result often is a change in the position of the peak transport in CAM3. As the transport change is more spread out in AMEP and also is not clearly dipolar, the total transport shifts up or down instead.

\section{Summary and discussion}

SST perturbations induce changes in the atmospheric general circulation and in storm tracks (Bengtsson et al. 2006; Brayshaw et al. 2008; Lu et al. 2010; GL12). Here we have examined whether such changes can be captured using a simplified energy balance model based on the principle of maximum entropy production (MEP), as 

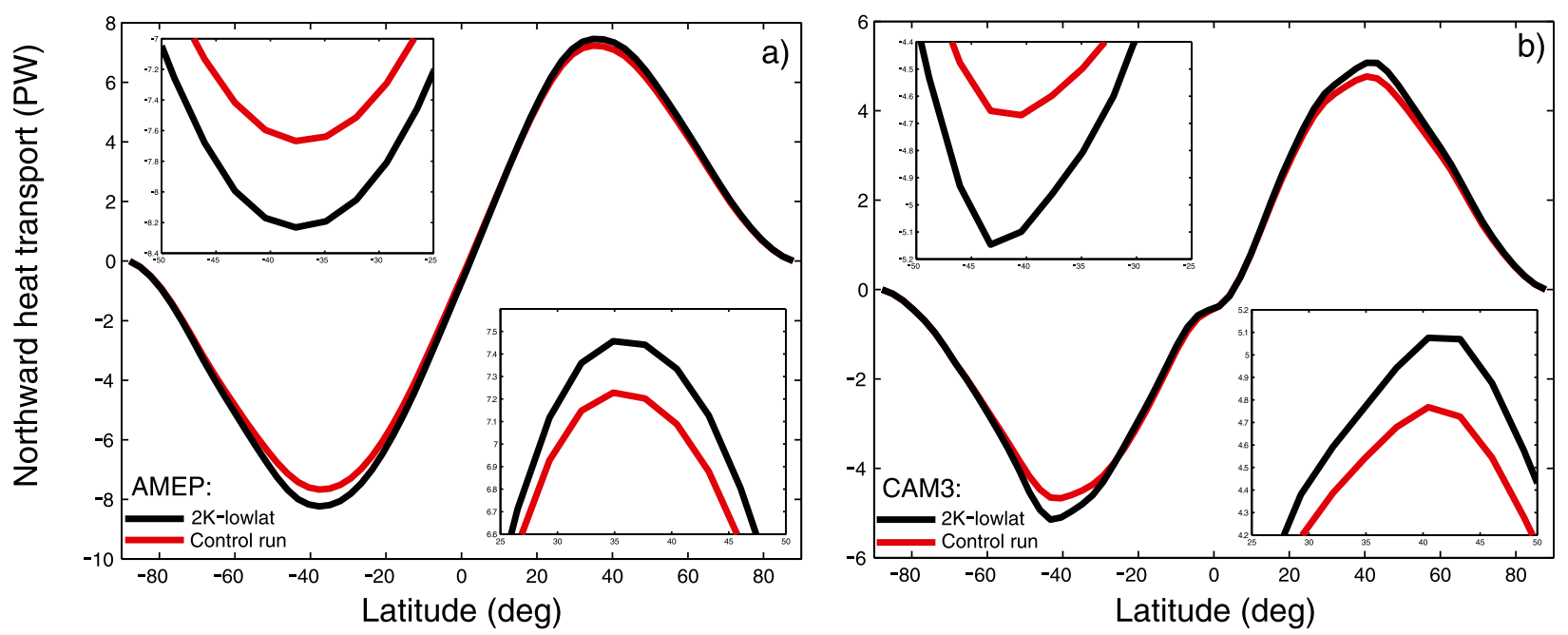

FIG. 12. Zonally averaged northward heat transport: control (red line) and 2K-lowlat (black line) solutions from (a) AMEP and (b) CAM3. Insets are close-ups of the maxima in the SH (upper-left corner) and NH (lower-right corner) transports.

such models have been shown previously to mimic aspects of Earth's climate system. To this end, we constructed an atmosphere-only EBM, called AMEP, and used it to study the response to changes in surface temperature.

AMEP has a single box in the vertical and thus represents the vertically and zonally averaged mean atmospheric temperature. Any number of boxes can be used; we chose 64 to yield the same resolution as with CAM3 (T42 resolution), the AGCM we used for comparison. Each box is heated by the sun and by convective fluxes from the surface. Energy balance yields a single equation with three unknowns: temperature, convective heat flux, and meridional heat flux.

To close the system, we impose two additional constraints: both vertical and lateral heat transport should maximize entropy production. We determine the convective heat flux first by imposing MEP and assuming zero lateral fluxes. This yields an expression for the convective flux in terms of the atmospheric and surface temperatures. We then use this to solve for the lateral fluxes in the horizontal, again using MEP.

The convective flux calculation produces a reasonable result. Using the MEP expression in (9) with the air and surface temperatures in CAM3, combined with a globalmean emissivity estimated from the CAM3 control run, yields convective fluxes very similar to those in the model. Further, the AMEP "control run" fluxes match well with those obtained in the CAM3 control run. The MEP solution performs poorly only at the equator, where the AGCM exhibits a distinct minimum.

AMEP also yields plausible temperature and meridional heat flux profiles, as compared to the control run with CAM3. The AMEP transports are somewhat larger, but the structure is similar. AMEP also exhibits qualitatively correct changes in response to surface temperature perturbations. Having a greater surface temperature increases the convective heating of the overlying box, which in turn increases the lateral heat flux out of the box. As such, the bulk of the changes occur in the latitudes where the perturbations are applied.

The response in CAM3, however, differs in several respects. The changes in convective heating are greater in most cases and are more localized near the forcing regions. This produces a stronger impact on the meridional heat fluxes, often shifting the position of the maximum heat transport (and hence the storm tracks). In AMEP, the less localized changes produce instead a broad increase or decrease in transport, so one would infer a change in storm-related variability, but not necessarily in the location of the storm track.

There are several reasons why AMEP differs on these points. For one, AMEP treats the tropics as it does the midlatitudes; meridional transport is assumed to be carried by turbulent eddies. AMEP lacks a Hadley circulation and so cannot simulate how that changes with increased surface heating ( $\mathrm{Lu}$ et al. 2007; GL12). As seen here, convective heating in CAM3 was 4 times larger in the tropics simulation, while also exhibiting the characteristic minimum at the equator. Both are related to the action of the Hadley cells.

A second point concerns the localization of the response to surface forcing. In AMEP, there are changes in convective flux of opposite sign outside the forcing range, and these are uniform with latitude. This occurs because excess heat is easily redistributed in AMEP. The localization seen in CAM3 implies that it is harder 
to spread heat. The storms in CAM3 result from baroclinic instability, which can be suppressed, for example, by the $\beta$ effect (e.g., Pedlosky 1982). No such conditions are at play in AMEP.

An additional reason concerns the emissivity. The changes in the lateral heat transport are strongly tied to those in convective heating in both models. The MEP convective flux [see (9)] is a strong function of emissivity. We took $\epsilon$ to be constant in these solutions, but in reality, it varies with temperature (Crawford and Duchon 1999; Iziomon et al. 2003). The mean emissivity was in fact slightly larger in the $2 \mathrm{~K}$-lowlat run, and using this value produces convective fluxes which are comparable to the observed (not shown). Thus, a potential improvement to the present model would be to take emissivity's temperature dependence into account when maximizing the lateral heat fluxes. However, increasing $\epsilon$ in the tropics case did not produce nearly large enough fluxes, so the lack of tropical dynamics remains an issue.

Nevertheless, many aspects in the CAM3 runs are captured in the AMEP model. AMEP performs particularly well when the forcing was applied at high latitudesmost likely because these runs do not entail changes in the tropics. This suggests that MEP is better at representing eddy transport at mid- and high latitudes than at the low latitudes.

The fact that AMEP partially succeeds suggests that changes in the storm track may well be a matter of redistributing excess heat. Moreover, we have seen that shifts in the storm tracks in CAM3 are often directly linked to dramatic changes in convective heating. Of course, latent and sensible heating depends on the airsea temperature difference, which itself is part of the solution. Convective heating is an additional source of heat which then must be transported laterally.

With regard to whether an AMEP-type model could be used for climate prediction, we note one further caveat. The surface warming in CAM3 represents a combination of changes over the ocean and land. The warmings are treated as imposed forcing in AMEP but in reality, they are a part of a solution, as with a coupled climate model. But, like an AGCM, AMEP has some value in understanding the atmospheric response.

Acknowledgments. We wish to thank Anne Fouilloux, Maarten H. P. Ambaum, Valerio Lucarini, and one anonymous reviewer for their insightful and clarifying comments, which greatly improved this manuscript.

\section{REFERENCES}

Bengtsson, L., K. I. Hodges, and E. Roeckner, 2006: Storm tracks and climate change. J. Climate, 19, 3518-3543, doi:10.1175/JCLI3815.1.
Blackmon, M. L., J. M. Wallace, N.-C. Lau, and S. L. Mullen, 1977: An observational study of the Northern Hemisphere wintertime circulation. J. Atmos. Sci., 34, 1040-1053, doi:10.1175/ 1520-0469(1977)034<1040:AOSOTN>2.0.CO;2.

Brayshaw, D. J., B. J. Hoskins, and M. Blackburn, 2008: The stormtrack response to idealized SST perturbations in an aquaplanet GCM. J. Atmos. Sci., 65, 2842-2860, doi:10.1175/ 2008JAS2657.1.

Busse, F. H., 1970: Bounds for turbulent shear flow. J. Fluid Mech., 41, 219-240, doi:10.1017/S0022112070000599.

Butler, A. H., D. W. J. Thompson, and T. Birner, 2011: Isentropic slopes, downgradient eddy fluxes, and the extratropical atmospheric circulation response to tropical tropospheric heating. J. Atmos. Sci., 68, 2292-2305, doi:10.1175/ JAS-D-10-05025.1.

Caldeira, K., 2007: The maximum entropy principle: a critical discussion. Climatic Change, 85, 267-269, doi:10.1007/ s10584-007-9335-3.

Chang, E. K. M., S. Lee, and K. L. Swanson, 2002: Storm track dynamics. J. Climate, 15, 2163-2183, doi:10.1175/ 1520-0442(2002)015<02163:STD > 2.0.CO;2.

Chen, G., J. Lu, and D. M. Frierson, 2008: Phase speed spectra and the latitude of surface westerlies: Interannual variability and global warming trend. J. Climate, 21, 5942-5959, doi:10.1175/ 2008JCLI2306.1.

Crawford, T. M., and C. E. Duchon, 1999: An improved parameterization for estimating effective atmospheric emissivity for use in calculating daytime downwelling longwave radiation. J. Appl. Meteor., 38, 474-480, doi:10.1175/1520-0450(1999)038<0474: AIPFEE $>2.0 . \mathrm{CO} ; 2$.

de Groot, S. R., and P. Mazur, 1963: Non-Equilibrium Thermodynamics. Dover Publications, 510 pp.

Dewar, R., 2003: Information theory explanation of the fluctuation theorem, maximum entropy production and self-organized criticality in non-equilibrium stationary states. J. Phys. A: Math. Gen., 36, 631-641, doi:10.1088/0305-4470/36/3/303.

Donohoe, A., and D. S. Battisti, 2011: Atmospheric and surface contributions to planetary albedo. J. Climate, 24, 4402-4418, doi:10.1175/2011JCLI3946.1.

Goody, R., 2007: Maximum entropy production in climate theory. J. Atmos. Sci., 64, 2735-2739, doi:10.1175/JAS3967.1.

Graff, L. S., and J. H. LaCasce, 2012: Changes in the extratropical storm tracks in response to changes in SST in an AGCM. J. Climate, 25, 1854-1870, doi:10.1175/JCLI-D-11-00174.1.

Grassl, H., 1981: The climate at maximum entropy production by meridional atmospheric and oceanic heat fluxes. Quart. J. Roy. Meteor. Soc., 107, 153-166, doi:10.1002/ qj.49710745110.

Grinstein, G., and R. Linsker, 2007: Comments on a derivation and application of the 'maximum entropy production' principle. J. Phys. A: Math. Theor., 40, 9717-9720, doi:10.1088/ 1751-8113/40/31/N01.

Hoskins, B. J., and P. J. Valdes, 1990: On the existence of storm-tracks. J. Atmos. Sci., 47, 1854-1864, doi:10.1175/ 1520-0469(1990)047<1854:OTEOST>2.0.CO;2.

Howard, L. N., 1972: Bounds on flow quantities. Annu. Rev. Fluid Mech., 4, 473-494, doi:10.1146/annurev.fl.04.010172.002353.

Iziomon, M. G., H. Mayer, and A. Matzarakis, 2003: Downward atmospheric longwave irradiance under clear and cloudy skies: Measurement and parameterization. J. Atmos. Sol. Terr. Phys., 65, 1107-1116, doi:10.1016/j.jastp.2003.07.007.

Kittel, C., and H. Kroemer, 1980: Thermal Physics. W. H. Freeman, 473 pp. 
Kleidon, A., 2004: Beyond Gaia: Thermodynamics of life and earth system functioning. Climatic Change, 66, 271-319, doi:10.1023/ B:CLIM.0000044616.34867.ec.

— 2009: Nonequilibrium thermodynamics and maximum entropy production in the earth system. Naturwissenschaften, 96, 653-677, doi:10.1007/s00114-009-0509-x.

Kushner, P. J., I. M. Held, and T. L. Delworth, 2001: Southern hemisphere atmospheric circulation response to global warming. J. Climate, 14, 2238-2249, doi:10.1175/1520-0442(2001)014<0001: SHACRT $>2.0 . \mathrm{CO} ; 2$.

Levitus, S., J. I. Antonov, T. P. Boyer, and C. Stephens, 2000: Warming of the world ocean. Science, 287, 2225-2229, doi:10.1126/science.287.5461.2225.

Lorenz, D. J., and E. T. DeWeaver, 2007: Tropopause height and zonal wind response to global warming in the IPCC scenario integrations. J. Geophys. Res., 112, D10119, doi:10.1029/2006JD008087.

Lorenz, E. N., 1955: Available potential energy and the maintenance of the general circulation. Tellus, 7,157-167, doi:10.1111/ j.2153-3490.1955.tb01148.x.

- 1960: Generation of available potential energy and the intensity of the general circulation. Dynamics of Climate, R. C. Pfeffer, Ed., Pergamon Press, 86-92.

Lorenz, R. D., 2002: Planets, life and the production of entropy. Int. J. Astrobiol., 1, 3-13, doi:10.1017/S1473550402001027.

— J. I. Lunine, P. G. Withers, and C. P. McKay, 2001: Titan, Mars and Earth: Entropy production by latitudinal heat transport. Geophys. Res. Lett., 28, 415-418, doi:10.1029/2000GL012336; Corrigendum, 28, 3169, doi:10.1029/2001GL013455.

Lu, J., G. A. Vecchi, and T. Reichler, 2007: Expansion of the Hadley cell under global warming. Geophys. Res. Lett., 34, L06805, doi:10.1029/2006GL028443.

- G. Chen, and D. M. W. Frierson, 2008: Response of the zonal mean atmospheric circulation to El Niño versus global warming. J. Climate, 21, 5835-5851, doi:10.1175/2008JCLI2200.1.

,,-- and D. M. Frierson, 2010: The position of the midlatitude storm track and eddy-driven westerlies in aquaplanet AGCMs. J. Atmos. Sci., 67, 3984-4000, doi:10.1175/2010JAS3477.1.

Lucarini, V., 2009: Thermodynamic efficiency and entropy production in the climate system. Phys. Rev. E, 80, 021118, doi:10.1103/PhysRevE.80.021118.

—_, and F. Ragone, 2011: Energetics of climate models: Net energy balance and meridional enthalpy transport. Rev. Geophys., 49, RG1001, doi:10.1029/2009RG000323.

Malkus, W. V. R., 1954: The heat transport and spectrum of thermal turbulence. Proc. Roy. Soc. London, A225, 1954-1954, doi:10.1098/rspa.1954.0197.

McCabe, G. J., M. P. Clark, and M. C. Serreze, 2001: Trends in Northern Hemisphere surface cyclone frequency and intensity. J. Climate, 14, 2763-2768, doi:10.1175/1520-0442(2001)014<2763: TINHSC $>2.0 . \mathrm{CO} ; 2$.

O'Brien, D. M., and G. L. Stephens, 1995: Entropy and climate. II: Simple models. Quart. J. Roy. Meteor. Soc., 121, 1773-1796, doi:10.1002/qj.49712152712.
Ozawa, H., and A. Ohmura, 1997: Thermodynamics of a global-mean state of the atmosphere-A state of maximum entropy increase. J. Climate, 10, 441-445, doi:10.1175/1520-0442(1997)010<0441: TOAGMS $>2.0 . \mathrm{CO} ; 2$.

,,-- R. D. Lorenz, and T. Pujol, 2003: The second law of thermodynamics and the global climate system: A review of the maximum entropy production principle. Rev. Geophys., 41, 1018, doi:10.1029/2002RG000113.

Paltridge, G. W., 1975: Global dynamics and climate-A system of minimum entropy exchange. Quart. J. Roy. Meteor. Soc., 101, 475-484, doi:10.1002/qj.49710142906.

_ 1978: The steady-state format of global climate. Quart. J. Roy. Meteor. Soc., 104, 927-945, doi:10.1002/qj.49710444206.

_- G. Farquhar, and M. Cuntz, 2007: Maximum entropy production, cloud feedback, and climate change. Geophys. Res. Lett., 34, L14708, doi:10.1029/2007GL029925.

Pascale, S., J. M. Gregory, M. Ambaum, and R. Tailleux, 2011: Climate entropy budget of the HadCM3 atmosphere-ocean general circulation model and of FAMOUS, its lowresolution version. Climate Dyn., 36, 1189-1206, doi:10.1007/ s00382-009-0718-1.

,-- M. H. P. Ambaum, R. Tailleux, and V. Lucarini, 2012: Vertical and horizontal processes in the global atmosphere and the maximum entropy production conjecture. Earth Syst. Dynam., 3, 19-32, doi:10.5194/esd-3-19-2012.

Pedlosky, J., 1982: Geophysical Fluid Mechanics. Springer-Verlag, $636 \mathrm{pp}$.

Polvani, L. M., and P. J. Kushner, 2002: Tropospheric response to stratospheric perturbations in a relatively simple general circulation model. Geophys. Res. Lett., 29, 1114, doi:10.1029/ 2001 GL014284.

Prigogine, I., 1947: Étude Thermodynamique des Phénomènes Irréversibles. Dunod, $143 \mathrm{pp}$.

Pujol, T., and J. E. Llebot, 2000b: Extremal climatic states simulated by a 2-dimensional model. Part II: Different climatic scenarios. Tellus, 52A, 440-454, doi:10.1034/ j.1600-0870.2000.00193.x.

Son, S.-W., and Coauthors, 2008: The impact of stratospheric ozone recovery on the Southern Hemisphere westerly jet. Science, 320, 1486-1489, doi:10.1126/science.1155939.

Swinbank, W. C., 1963: Long-wave radiation from clear skies. Quart. J. Roy. Meteor. Soc., 89, 339-348, doi:10.1002/ qj.49708938105.

Wang, X. L., V. R. Swail, and F. W. Zwiers, 2006: Climatology and changes of extratropical cyclone activity: Comparison of ERA-40 with NCEP-NCAR reanalysis for 1958-2001. J. Climate, 19, 3145-3166, doi:10.1175/JCLI3781.1.

Yin, J. H., 2005: A consistent poleward shift of the storm tracks in simulations of 21 st century climate. Geophys. Res. Lett., 32, L18701, doi:10.1029/2005GL023684.

Ziegler, H., and C. Wehrli, 1987: On a principle of maximal rate of entropy production. J. Non-Equilib. Thermodyn., 12, 229-244, doi:10.1515/jnet.1987.12.3.229. 